\title{
Channel Estimation and Signal Detection for Multi-Carrier CDMA Systems with Pulse-Shaping Filter
}

\author{
Mohammad Jaber Borran, Prabodh Varshney, Hannu Vilpponen, and Panayiotis D. Papadimitriou \\ Nokia Mobile Phones, 6000 Connection Drive, 2:200, Irving, TX 75039 \\ Email: mohammad@rice.edu, \{prabodh.varshney,hannu.vilpponen,panayiotis.papadimitriou\}@nokia.com
}

\begin{abstract}
We consider the problem of digital communication in a fading environment using the Multi-Carrier CDMA technology. By incorporating the effect of the pulse-shaping filter in the channel estimation and signal detection modules, we develop new estimator and detector structures which significantly outperform the commonly used time-domain equalizers and matched filter detector. Our simulation results demonstrate the elimination of some of the error floors which one would experience if usual time-domain techniques were used to cancel the effect of nonideal pulse-shaping filter.
\end{abstract}

Index Terms-Multi-carrier CDMA systems, OFDM, pulseshaping filter, channel estimation, multiuser detection, wireless communications

\section{INTRODUCTION}

The Multi-Carrier Code-Division Multiple Access (MCCDMA) technology [1], [2] has been proposed to combat the multipath and to use frequency selectivity of the channel as a means of diversity (frequency diversity). By dividing the total users into smaller blocks of few users in each, this system also permits the use of short spreading sequences and Maximum Likelihood (ML) detection inside each block with a reasonable computational complexity. Different channel estimation and signal detection techniques for Orthogonal Frequency-Division Multiplexing (OFDM) and MC-CDMA systems have been studied in [1]-[6]. The main problem arises when a predefined (e.g. by a standard) pulse-shaping filter has to be applied before transmission. These filters are usually truncated sampled versions of continuous-time lowpass filters, and often do not have the ideally desired impulse and frequency responses, i.e., they introduce Inter-Symbol Interference (ISI), and are not completely flat in the passband. Using the same filter at the receiver as a matched filter is optimal only if the composite filter is ISI-free, e.g., if each filter is a square-root raised cosine filter. This is not necessarily true for all cases, e.g., the pulseshaping filter specified in the IS-95 standard (which is also later adopted by other standards such as CDMA2000) does not have this property. In order to completely cancel the effect of this pulse-shaping filter in time domain at the receiver, one could use a Minimum Mean-Square Error (MMSE) equalizer or a maximum likelihood sequence estimator (depending on the type of the desired optimization, i.e., continuous vs. discrete), or equivalently, consider the filter as part of the channel and equalize for the composite channel. This would, however, significantly increase the complexity of the equalizer, because the memory of the channel would increase by the length of the pulse-shaping filter. For example, the pulse-shaping filter of IS-95 spans over 12 chips (assuming a chip period of around $813.8 \mathrm{nsec}$ ). If the same filter is used at the receiver, and if the delay spread of the channel is around $5 \mu \mathrm{sec}$, then the composite channel will have a total memory of around 30 chips, as compared to the actual channel which has a memory of only 6 chips. Even after discarding the small coefficients of the composite channel, the complexity of the equalizer or sequence estimator for the composite channel will be significantly larger than the one for the original channel.

In this paper, we propose a method to cancel the effect of the pulse-shaping filter in frequency domain. The new method significantly improves the performance of the system as compared to using the same filter at the receiver and assuming an ISI-free composite filter. The paper is organized as follows. Section II presents the system model considered throughout the paper. Section III discusses an upsampling and downsampling method using Inverse and direct Fast Fourier Transform (IFFT and FFT). Sections IV and V introduce the proposed channel estimation and data detection techniques. Section VI presents the results of our simulations and compares the performance of the proposed system with a system which only uses the same pulse-shaping filter at the receiver without any further processing. The main idea and the results of the paper are summarized in Section VII.

\section{SySTEM MOdEL}

The block diagram of the baseband MC-CDMA system [2] is shown in Fig. 1. The modulated symbol streams of all of the users are split into $K$ blocks of $J$ streams each. Inside each block, the streams are spread using Walsh-Hadamard codes of length $N$, and then summed up to form a single spread stream. The spread streams at the outputs of the blocks are then passed through a serial to parallel converter, and then 


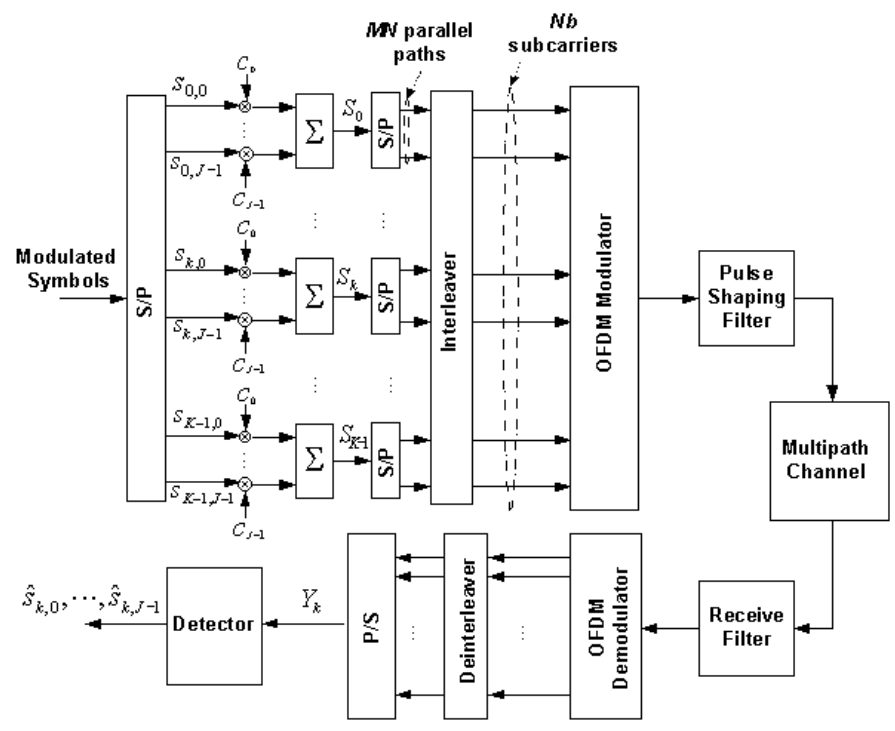

Fig. 1. Block diagram of the MC-CDMA system.

interleaved and mapped into $N_{b}$ different frequency bins using an OFDM modulator (IFFT). The output of IFFT is assumed to be of length $T$ seconds in time, resulting in a chip interval of $T_{c}=\frac{T}{N_{b}}$. A cyclic prefix of appropriate length is then added to the time-domain signal at the output of IFFT to prevent ISI and ICI (Inter-Symbol and Inter-Channel Interferences). This time-domain signal is then passed through the pulse-shaping filter and transmitted over the channel.

At the receiver, the signal is first passed through a bandpass receive filter (this can be same as the pulse-shaping filter, or more accurately, a filter matched to the pulse-shaping filter) to suppress the out-of-band noise and interference. After the receive filter, the signal is passed through an OFDM demodulator (FFT) and then deinterleaved and converted to serial to form the spread streams corresponding to the transmitter block outputs. Inside each block at the receiver, a multi-user detector is used to generate soft or hard decision outputs for each original symbol or bit stream.

We denote the Walsh-Hadamard codes by vectors $c_{0}, \cdots, c_{J-1}$ of length $N$, and the symbol streams at the inputs of the $k$ th block by vectors $s_{k, 0}, \cdots, s_{k, J-1}$ of length $M$, where $M=\frac{N_{b}}{K N}$. Then the spread stream at the output of this block will be a vector of length $M N$, given by

$$
S_{k}(m N+n)=\sum_{j=0}^{J-1} s_{k, j}(m) c_{j}(n),
$$

for $k=0, \cdots, K-1, m=0, \cdots, M-1$, and $n=0, \cdots, N-$ 1 . We use the notation $v(p)$ to denote the $p$ th element of the vector $v$. The output of the interleaver is a vector $X$ of length $N_{b}$, which is constructed from elements of $S_{0}, \cdots, S_{K-1}$, but with a new order.

We assume that all of the time-domain signals, as well as the channel and filter impulse responses, are discretized at a sampling rate of $N_{s}$ samples per chip ( $\frac{N_{s}}{T_{c}}$ samples per second), and denote the upsampled version of the time-domain signal by vector $x$. We also assume that a cyclic prefix of length $N_{c} N_{s}$ samples has been added to the beginning of this vector.

We further assume that the channel is a multipath channel of length $L_{c}$ samples, and the pulse-shaping filter is of length $L_{t}$ samples. If we denote the impulse responses of the channel and transmit pulse shaping filter by $h_{c}$ and $h_{t}$, the received time-domain signal at the input of the receiver will be given by

$$
y(i)=\sum_{l_{c}=0}^{L_{c}-1} h_{c}\left(l_{c}\right) \sum_{l_{t}=0}^{L_{t}-1} h_{t}\left(l_{t}\right) x\left(i-l_{c}-l_{t}\right)+\nu(i),
$$

for $i=0, \cdots,\left(N_{c}+N_{b}\right) N_{s}-1$, where $\nu(i)$ is a zero-mean additive white Gaussian noise of variance $\sigma^{2}$.

At the receiver, the received signal is first passed through a lowpass filter to suppress the out-of-band noise and interference (as we will see later, this filter can have a wider bandwidth than the transmit filter and offer better frequency response in the passband), and then after discarding the cyclic prefix, downsampled and passed through an OFDM demodulator (FFT). We denote this frequency-domain signal by vector $Z$.

Assuming that the receive filter is FIR of length $L_{r}$ and that the cyclic prefix is long enough (i.e., longer than $L_{r}+L_{c}+$ $L_{t}-2$ ), we can write

$$
Z=H_{r} H_{c} H_{t} X+\mathcal{N}_{Z},
$$

where $H_{r}, H_{c}$, and $H_{t}$ are diagonal matrices constructed from the frequency components of the receive filter, channel, and transmit pulse-shaping filter in the desired band, and $\mathcal{N}_{Z}$ is the filtered noise in frequency domain. Since the frequency response of the receive filter is not necessarily flat in the passband, entries of $\mathcal{N}_{Z}$ do not have the same variance (they are still independent, though). In order to obtain an i.i.d. noise term, we can divide the entries of vector $Z$ by corresponding frequency components of the received filter. We denote the resulting vector by $Y$,

$$
Y=H_{r}^{-1} Z=H_{c} H_{t} X+\mathcal{N},
$$

where entries of $\mathcal{N}$ are i.i.d. In the rest of this paper, we will use this system model for channel estimation and signal detection.

\section{UPSAMPLING AND DOWNSAMPLING USING FFT}

In order to generate a time-domain transmit signal at the sampling rate of $N_{s}$ samples per chip, one option would be taking an IFFT of size $N_{b}$ at the transmitter, and performing the upsampling by inserting $N_{s}-1$ zeros between each pair of consecutive time-domain chips and passing the resulting signal through the pulse-shaping filter. If the pulse-shaping filter was an ideal lowpass filter, this approach would be optimal, i.e., equivalent to reconstructing the continuous-time signal and resampling it at this higher sampling rate. However, as mentioned 
earlier, because of the truncation in time and other practical considerations, the pulse-shaping filters are not usually ideal. A more accurate approach to construct the upsampled version of the time-domain signal is to use a larger IFFT. For this, we split the input vector of IFFT, $X$, into two parts of length $N_{b} / 2$ each, and insert $N_{b}\left(N_{s}-1\right)$ zeros between them (i.e., at high frequencies), to form a new vector of length $N_{b} N_{s}$. Then we take an IFFT of size $N_{b} N_{s}$ to obtain a time-domain signal of the same length. This way, we have generated a timedomain signal of the desired sampling rate without modifying the frequency content of the signal.

At the receiver, we would like to have $N_{b}$ symbols at the output of the OFDM demodulator, corresponding to the $N_{b}$ transmitted symbols, $X$. One way to obtain these $N_{b}$ symbols in the frequency domain would be downsampling (decimating) the output of the receive filter (after removing the cyclic prefix), and then taking an FFT of size $N_{b}$. Similar to the argument for the transmitter side, however, a more accurate frequencydomain signal can be obtained by taking an FFT of size $N_{b} N_{s}$, and then discarding the high frequency components (middle $N_{b}\left(N_{s}-1\right)$ symbols) and constructing the desired signal by merging the low-frequency parts $\left(N_{b} / 2\right.$ symbols at the two ends). This operation is itself a lowpass filtering (carried out in the frequency domain), and can replace the receive filter if the bandwidth of the received time-domain signal is not more than $\frac{N_{s}}{T_{c}} \mathrm{~Hz}$. Therefore, the receive filter can be $N_{s}$ times wider than the transmit pulse-shaping filter, making it possible to design filters which have smaller ripples in the desired band.

The above upsampling and downsampling methods, together with the appropriate channel estimation and detection methods explained in the next two sections, result in a significant improvement in the performance as compared to the conventional methods.

\section{Channel Estimation}

We use a channel estimation technique similar to [5] and [6]. As suggested in [5], we use equally spaced pilot symbols in frequency domain. The minimum number of the required pilot symbols is equal to the length of the impulse response of the channel. One option is to consider $H_{c} H_{t}$ as a composite channel. Estimating this channel would require at least $L_{c}+$ $L_{t}-1$ pilots. However, we can use the fact that $H_{t}$ is known, and estimate only $H_{c}$, which would require a minimum of $L_{c}$ pilots. Therefore, we derive the channel estimator using this fact.

We assume that the transmitted frequency-domain pilot symbols are all ones. We collect, in vector $Y_{p}$, those elements of $Y$ which correspond to pilot symbols, and similarly construct the vectors $H_{c p}$ and $\mathcal{N}_{p}$ and the matrix $H_{t p}$ from the corresponding elements of $H_{c}, \mathcal{N}$, and $H_{t}$, respectively. Now, using (4), and restricting ourselves to only pilot symbols, we will have

$$
Y_{p}=H_{t p} H_{c p}+\mathcal{N}_{p}
$$

Denoting the matrix consisting of the first $L_{c}$ columns of the FFT matrix by $W$, we have

$$
H_{c}=\operatorname{diag}\left(W h_{c}\right)
$$

where $\operatorname{diag}(v)$ denotes the diagonal matrix whose diagonal elements are elements of vector $v$. If we further restrict ourselves to only those rows where pilot symbols were transmitted, and denote the resulting FFT matrix by $W_{p}$, we will have

$$
H_{c p}=W_{p} h_{c}
$$

Substituting (7) in (5), we will have

$$
Y_{p}=H_{t p} W_{p} h_{c}+\mathcal{N}_{p}
$$

Now, the ML estimate for $h_{c}$ is obtained by maximizing the log-likelihood function

$$
L\left(h_{c}\right)=\ln p\left(Y_{p} \mid h_{c}\right)=A-B\left\|Y_{p}-H_{t p} W_{p} h_{c}\right\|^{2},
$$

where $A$ and $B$ are constant scalars and do not depend on $h_{c}$, and $B>0$. Therefore, the estimation problem is equivalent to the following optimization problem

$$
\widehat{h}_{c}=\arg \min _{h_{c}}\left\|Y_{p}-H_{t p} W_{p} h_{c}\right\|^{2} .
$$

The optimization in (10) is, in fact, a least squares (LS) problem, and the solution is obtained as follows

$$
\widehat{h}_{c}=\left(W_{p}^{H} H_{t p}^{H} H_{t p} W_{p}\right)^{-1} W_{p}^{H} H_{t p}^{H} Y_{p} .
$$

In practice, the condition number of the matrix $W_{p}^{H} H_{t p}^{H} H_{t p} W_{p}$ can become very large, making the above solution unstable. Therefore, it is preferable to use the more stable method of Singular Value Decomposition (SVD) for solving the LS problem. If the SVD of $H_{t p} W_{p}$ is given by

$$
H_{t p} W_{p}=U S V^{H},
$$

the solution to the LS problem (10) will be given by

$$
\widehat{h_{c}}=V S^{-1} U^{H} Y_{p} .
$$

(If some of the singular values are equal to zero, the corresponding elements in $S^{-1}$ in the above equation should be replaced by zeros.)

After estimating $h_{c}$, the frequency response of the channel can be obtained by taking the FFT of $\widehat{h}_{c}$,

$$
\widehat{H}_{c}=\operatorname{diag}\left(W \widehat{h}_{c}\right)=\operatorname{diag}\left(W V S^{-1} U^{H} Y_{p}\right) .
$$

Notice that all of the matrices $W, H_{t}, V, S$, and $U$, are known a priori. Therefore, the matrix $L=W V S^{-1} U^{H}$ can be calculated offline, and for each OFDM symbol, the channel estimation in (14) will simply be a matrix-by-vector multiplication, $\widehat{H}_{c}=\operatorname{diag}\left(L Y_{p}\right)$. 


\section{Detection}

Once we have the channel estimate, $\widehat{H}_{c}$, we combine this estimate and the frequency response of the transmit pulseshaping filter, $H_{t}$, in a single composite channel matrix, $\mathcal{H}=H_{c} H_{t}$. If we denote the outputs of the deinterleaver corresponding to the block outputs $S_{k}$, by vector $Y_{k}$, and the corresponding composite channel and additive noise values by $\mathcal{H}_{k}$ and $\mathcal{N}_{k}$, then from (4) we will have

$$
Y_{k}=\mathcal{H}_{k} S_{k}+\mathcal{N}_{k}
$$

Different detection techniques can then be applied to this model to obtain the transmitted symbol streams $s_{k, j}$. Since the transmitted symbols are assumed to be independent, we can detect one symbol from each stream at a time. Therefore, we further divide (15) into smaller pieces, each one containing only one symbol from each stream. For that, we denote those elements of $Y_{k}, \mathcal{H}_{k}, S_{k}$, and $\mathcal{N}_{k}$ corresponding to the $m$ th transmitted symbol from each stream, by $Y_{k, m}, \mathcal{H}_{k, m}, S_{k, m}$, and $\mathcal{N}_{k, m}$, respectively, for $m=0, \cdots, M-1$. For example, $S_{k, m}=\left[S_{k}(m N), \cdots, S_{k}(m N+N-1)\right]^{T}$. With this notation, from (1) we will have

$$
\begin{aligned}
Y_{k, m} & =\mathcal{H}_{k, m} S_{k, m}+\mathcal{N}_{k, m} \\
& =\mathcal{H}_{k, m} \sum_{n=0}^{N-1} s_{k, n}(m) c_{n}+\mathcal{N}_{k, m}
\end{aligned}
$$

for $k=0, \cdots, K-1$, and $m=0, \cdots, M-1$, and the goal will be to find $s_{k, j}(m)$ for $j=0, \cdots, J-1$.

\section{A. Conventional (Matched Filter) Detector}

The conventional or Matched Filter (MF) detector first cancels the effect of the composite channel by dividing $Y_{k, m}$ by $\mathcal{H}_{k, m}$, and then despreads the resulting stream with different spreading codes to obtain the following estimate for the transmitted symbol

$$
\widehat{s}_{k, j, M F}(m)=c_{j}^{H} \mathcal{H}_{k, m}^{-1} Y_{k, m} .
$$

This value, then, can be used to find the hard decisions or soft decisions on the transmitted symbols or bits. However, this approach is not optimal even though the Walsh-Hadamard spreading codes are orthogonal and the multi-access interference appears to have been completely cancelled by the above despreading. The reason is that after multiplying $Y_{k, m}$ by $\mathcal{H}_{k, m}^{-1}$, the noise terms will no longer be i.i.d., and simple despreading will not be optimal.

\section{B. Maximum Likelihood Detector}

The optimal detector will maximize the log-likelihood function, or equivalently solve the following optimization problem

$$
\min _{s_{k, 0}(m), \cdots, s_{k, J-1}(m)}\left\|Y_{k, m}-\mathcal{H}_{k, m} \sum_{j=0}^{J-1} s_{k, j}(m) c_{j}\right\|^{2} .
$$

The computational complexity of the above discrete optimization is exponential in $J, \mathcal{O}\left(2^{R J}\right)$, where $R$ is the rate in bits/symbol ( $2^{R}$ is the constellation size). However, if the spreading codes are real, for certain types of constellations, such as 16QAM, we can decouple the above problem into two disjoint optimizations on the real and imaginary parts of the constellation points. For this, we should first remove the phase of the channel,

$$
\widetilde{Y}_{k, m}=\exp \left(-j \angle \mathcal{H}_{k, m}\right) Y_{k, m} .
$$

If we denote the real and imaginary parts of $\tilde{Y}_{k, m}$ by $\tilde{Y}_{k, m}^{I}$ and $\tilde{Y}_{k, m}^{Q}$, respectively, and similarly the real and imaginary parts of the transmitted vectors by $s_{k, j}^{I}$ and $s_{k, j}^{Q}$, respectively, the above optimization problem will reduce to the following two problems

$$
\begin{array}{r}
\min _{s_{k, 0}^{I}(m), \cdots, s_{k, J-1}^{I}(m)}\left\|\tilde{Y}_{k, m}^{I}-\left|\mathcal{H}_{k, m}\right| \sum_{j=0}^{J-1} s_{k, j}^{I}(m) c_{j}\right\|^{2}, \\
\min _{s_{k, 0}^{Q}(m), \cdots, s_{k, J-1}^{Q}(m)}\left\|\tilde{Y}_{k, m}^{Q}-\left|\mathcal{H}_{k, m}\right| \sum_{j=0}^{J-1} s_{k, j}^{Q}(m) c_{j}\right\|^{2} .
\end{array}
$$

The computational complexity of each one of the above optimizations is $\mathcal{O}\left(2^{R J / 2}\right)$. For example, for a 16QAM constellation, if $J=4$, the original optimization would require 65536 comparisons to detect (make hard decisions on) one symbol from each stream (4 symbols overall), whereas the simplified optimizations of (20) would require only 256 comparisons each.

\section{Simulation Results}

In this section, we present the results of our simulations for two systems which we will refer to as System A and System B. Both systems are MC-CDMA systems operating in $1.9 \mathrm{Ghz}$ band with 512 sub-carriers (OFDM bins) of width $2.494 \mathrm{Khz}\left(N_{b}=512, T=401 \mu \mathrm{sec}\right)$, and spreading factor of $4(N=4)$. In both systems, 16 equally spaced pilot symbols are inserted in each OFDM block (in frequency domain), and a cyclic prefix of length 20 chips is added to the beginning of each OFDM symbol. The pulse-shaping filter and the receive filter for both systems are the pulse-shaping filter specified in the IS-95 and CDMA2000 standards (we resampled that filter with the new sampling rate of $195.8 \mathrm{nsec}$ to match with the sample interval of our MC-CDMA systems). System A assumes that the composite filter (combination of the pulse shaping filter and the receive filter) is ISI-free, and does not perform any further processing to cancel the effect of those filters. The upsampling and downsampling for this system is done using the conventional methods (first method explained in Section III). System B exploits all of the techniques presented in this paper to cancel the effect of the pulse-shaping filter. The upsampling and downsampling are done using IFFT and FFT, and the channel estimation and signal detection modules use the knowledge of the frequency response of the filter, as explained in Sections IV and V. We ran the simulations for 


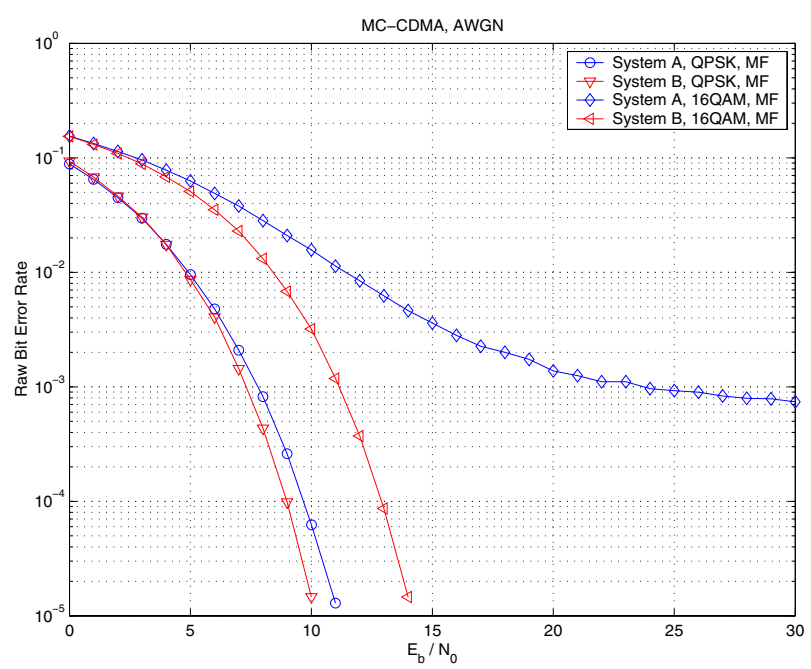

Fig. 2. Performance comparison in AWGN channel.

QPSK and 16QAM modulations in the AWGN channel and the ITU Vehicular A channel with $120 \mathrm{Km} / \mathrm{h}$. In the channel estimation module, we assumed $L_{c}=1$ or $L_{c}=8$ for the AWGN or Vehicular A channels, respectively. We compared the raw bit error rates of the two systems and the results are shown in Figures 2-4.

Fig. 2 presents the results for the AWGN channel. As expected, for both modulations, System B achieves better performance than System A. It is interesting to notice that System A with 16QAM, even in the AWGN channel, reaches an error floor of around $6 \times 10^{-4}$, whereas System B does not see any error floor in the AWGN channel. Fig. 3 presents the simulation results for QPSK modulation in the Vehicular A channel. As we see, system B with MF detector achieves a similar performance to System A with ML detector, and System B with ML detector achieves a much better performance than System A with either detector. Fig. 4 shows similar results for the 16QAM modulation in the Vehicular A channel.

\section{CONCLUSIONS}

We presented new techniques to deal with the effects of non-ideal pulse-shaping filters in MC-CDMA systems. We demonstrated upsampling and downsampling methods using IFFT and FFT. We also derived the channel estimation and signal detection techniques which exploit the knowledge of the frequency response of the pulse-shaping filter to improve the reliability of the channel estimates and performance of the detectors. Our simulation results show that the new techniques can result in significant improvement in the performance of the MC-CDMA systems in different channels as compared to the conventional systems which do not remove the residual degrading effects of the pulse-shaping filter.

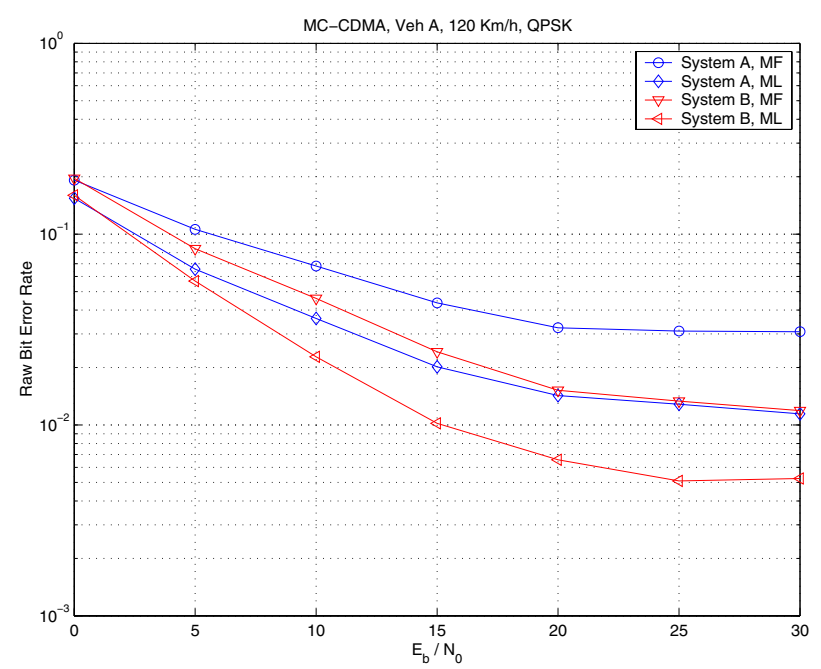

Fig. 3. Performance comparison for QPSK in Vehicular A channel.

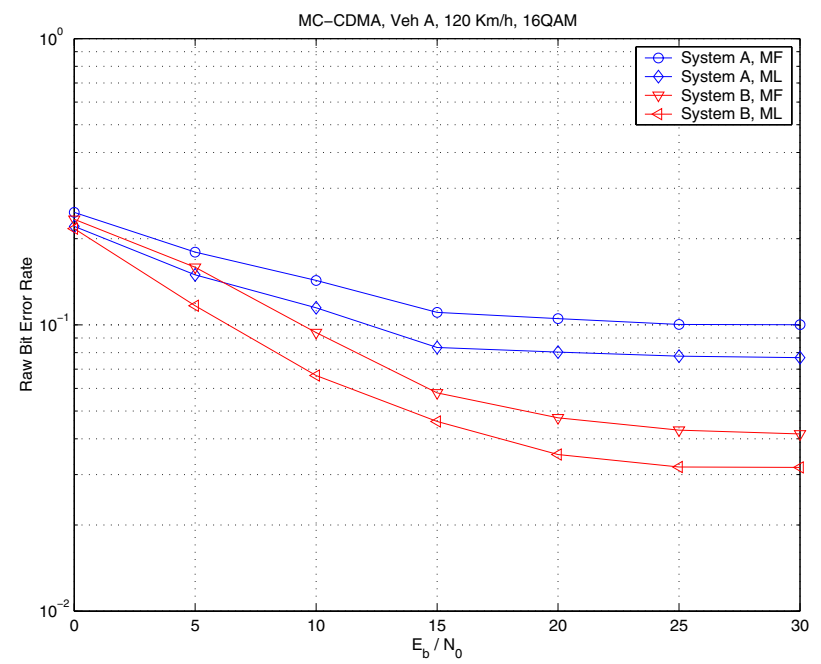

Fig. 4. Performance comparison for 16QAM in Vehicular A channel.

\section{REFERENCES}

[1] K. Fazel, "Performance of CDMA/OFDM for mobile communication system," in Conference Record, 2nd. International Conference on Universal Personal Communications, 1993, pp. 975-979.

[2] S. Kaiser, "OFDM-CDMA versus DS-CDMA: Performance evaluation for fading channels," in Proceedings of the International Conference on Communications, 1995, pp. 1722-1726.

[3] J. J. van de Beek, O. Edfors, and M. Sandell, "On channel estimation in OFDM systems," in Proceedings of the Vehicular Technology Conference, 1995, pp. 815-819.

[4] M. Hsieh and C. Wei, "Channel estimation for OFDM systems based on comb-type pilot arrangement in frequency selective fading channels," IEEE Transactions on Consumer Electronics, vol. 44, no. 1, pp. 217-225, Feb. 1998.

[5] R. Negi and J. Cioffi, "Pilot tone selection for channel estimation in a mobile OFDM system," IEEE Transactions on Consumer Electronics, vol. 44, no. 3, pp. 1122-1128, Aug. 1998.

[6] I. Barhumi, G. Leus, and M. Moonen, "Optimal training sequences for channel estimation in MIMO OFDM systems in mobile wireless channels," in Proceedings of the International Zurich Seminar on Broadband Communications, 2002, pp. 441-446. 